Methodology Primary studies were identified by following a defined search strategy on the prevalence of co-morbidity and survival pattern among ovarian cancer patients. This study has been conducted in accordance with PRISMA guidelines for systematic review. Co-morbidity assessment in the included studies had been done through the Charlson Co-morbidity Index (CCI) tool. Qualitative summarization of data from included studies for prevalence of various co-morbidities and influence of CCI score on survival in ovarian cancer patients has been performed.

Results Common co-morbidities prevalent in ovarian cancer patients were hypertension ( $11 \%$ to $26 \%)$, cardio vascular disease $(4.5 \%$ to $12 \%)$ and diabetes $(2.5 \%$ to $8.3 \%)$. Less commonly occurring co-morbidities were liver disease, renal disease, neurological problems and collagen vascular disease. Majority of ovarian cancer patients lie in CCI score $0(68 \%$ $76 \%)$. The range for one year $\%$ survival for CCI score 0 was 73 to $80 \%$, for CCI score $1-2$ : 58 to $71 \%$ and CCI score 2 $+: 43$ to $53 \%$. The range five year\% survival for CCI score 0 was 37 to $43 \%$, for CCI score $1-2$ : 24 to $30 \%$ and CCI score $2+: 12$ to $23 \%$.

Conclusion Co-morbidities plays an important role in survival outcomes among ovarian cancer patients. Overall one year\% and five year $\%$ survival decreases with increase in the CCI index score.

Disclosures The authors have no conflict of interest.

\section{COMPLETE SURGICAL CYTOREDUCTION REMAINS PIVOTAL IN ACHIEVING BETTER SURVIVAL OUTCOMES IN PATIENTS WITH ADVANCED OVARIAN CANCER WITH A BRCA1/2 GERMLINE MUTATION IN THE PRE-PARP INHIBITOR ERA}

Mohamed Otify, Alexandros Laios, Hannah Elkadi, Angelika Kaufmann, Sarika Munot, George Theophilou, Amudha Thangavelu, David Nugent, Tim Broadhead, Richard Hutson, Diderick De Jong. St James's University Hospital; Leeds Teaching Hospitals; Gynaecologic Oncology

\subsection{6/ijgc-2020-ESG0.120}

Introduction/Background Ovarian malignancies arising in patients with germline BRCA1/2 mutations are characterised by increased platinum sensitivity. It is not known if this feature should be considered when choosing between primary surgical and neoadjuvant therapy at the time of diagnosis. We hypothesised that patients with BRCA $1 / 2$ germline mutations have a better response to neoadjuvant chemotherapy, and their surgery may be less extended to achieve a complete surgical cytoreduction. We aimed to compare pre-operative, intra-operative, and survival parameters between patients with and without a BRCA $1 / 2$ germline mutation.

Methodology A total of 168 prospectively registered BRCA 1/ 2 typed women with a diagnosis of advanced ovarian cancer (AOC) at St James's Institute of Oncology, Leeds, who underwent cytoreductive surgery following neoadjuvant chemotherapy between October 2013 and October 2018 were identified. Primary outcomes included progression-free survival (PFS) and overall survival (OS). Secondary outcomes included chemotherapy response score (CRS), performance status (PS) and residual disease (RD). Differences between BRCA-positive and BRCA-negative groups were analysed using Stata $13^{\circledR}$. P $<0.05$ was considered as statistically significant.
Results Patients with a complete surgical cytoreduction had longer OS compared to those with an optimal or sub-optimal cytoreduction (48 vs 31 months, $p=0.0001$ ). PS and CRS were independent predictors for PFS, irrespective of BRCA status $(p=0.00001$ and 0.00006$)$. There was no significant difference between BRCA mutation carriers and non-carriers for mean PFS and OS (19 vs 18 months, and 50 vs 42 months, $\mathrm{p}=0.69$ and 0.39 , respectively). BRCA mutation carriers had no better chemo response or less extended surgery to achieve a complete surgical cytoreduction following neoadjuvant chemotherapy compared to non-carriers $(\mathrm{p}=0.67$ and 0.5 , respectively). In the subgroups of patients with a PS of 2 and those receiving single-agent chemotherapy, BRCA mutation carriers had shorter OS than non-carriers (RR 0.4 and 0.31 , 95\% CI $0.17-0.91$ and $0.1-0.93, p=0.029$ and 0.037 , respectively). In the subgroup of patients with a CRS of 3, BRCA mutation carriers had longer PFS and OS than non-carriers (RR 12.5 and 20.8 , 95\% CI 3.32-47.6 and 4.8-88.8, $\mathrm{p}=0.00$ and 0.00 , respectively).

Conclusion Complete surgical cytoreduction remains pivotal in achieving better survival outcomes in AOC women, irrespective of BRCA status. A survival benefit is unlikely for patients with poor PS, and those receiving single-agent chemotherapy. A favourable response to neoadjuvant chemotherapy or less extended surgery to achieve a complete surgical cytoreduction in patients with a BRCA $1 / 2$ germline mutation could not be confirmed.

Disclosures No Conflict of interest or any disclosure to make.

\section{TWO-YEAR PROGNOSIS ESTIMATION OF ADVANCED HIGH GRADE SEROUS OVARIAN CANCER PATIENTS USING MACHINE LEARNING}

${ }^{1}$ Alexandros Laios, ${ }^{2}$ Angeliki Katsenou, ${ }^{1}$ Yong Tan, ${ }^{1}$ Mohamed Otify, ${ }^{1}$ Angelika Kaufmann, ${ }^{1}$ Amudha Thangavelu, 'David Nugent, 'Diederick Dejong. 'St James's University Hospital: Leeds Teaching Hospitals; Gynaecologic Oncology; ${ }^{2}$ Visual Information Lab, University of Bristol; Department of Electrical and Electronic Engineering

\subsection{6/ijgc-2020-ESGO.121}

Introduction/Background Accurate prediction of patient prognosis can be especially useful for the selection of best treatment protocols. Machine Learning (ML) can serve this purpose by making predictions based upon generalizable clinical patterns embedded within learning datasets. We hypothesised that use of ML algorithms could improve prognosis estimation in advanced high grade serous ovarian (HGSOC) patients. We aimed to compare the performance of two ML prediction methods for HGSOC prognosis, based on Area Under Curve (AU-ROC) performance for a 2-year prognosis period.

Methodology This was a retrospective analysis of 209 FIGO stage III-IV HGSOC women, who were scheduled for cytoreductive surgery in SJUH, Leeds between Jan 2015 to Dec 2018 with curative or life-prolonging intent. Support-VectorMachine (SVM) and K-Nearest Neigbors (K-NN) were employed to model prognosis. The prognosis estimation problem was formulated as a binary classification problem. For the 2-year prognosis period, two groups were defined using patient survival information; patients who did not relapse or survived beyond two years were labelled in the positive class and patients who relapsed or died before reaching that period 
2-year progression free survival

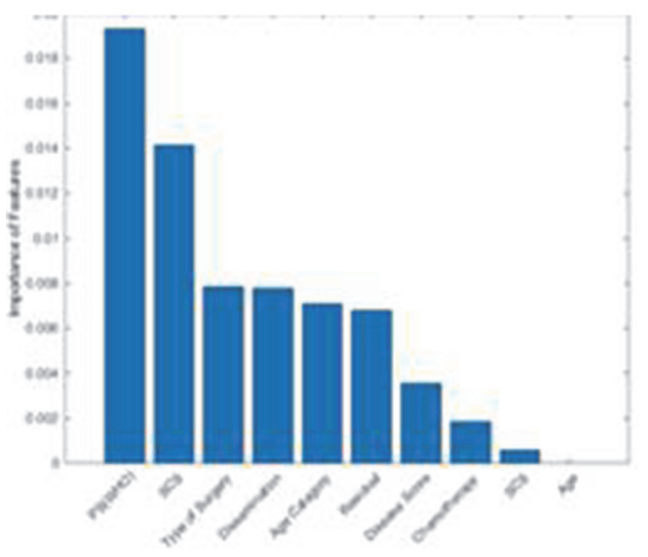

2-year overallsurvival

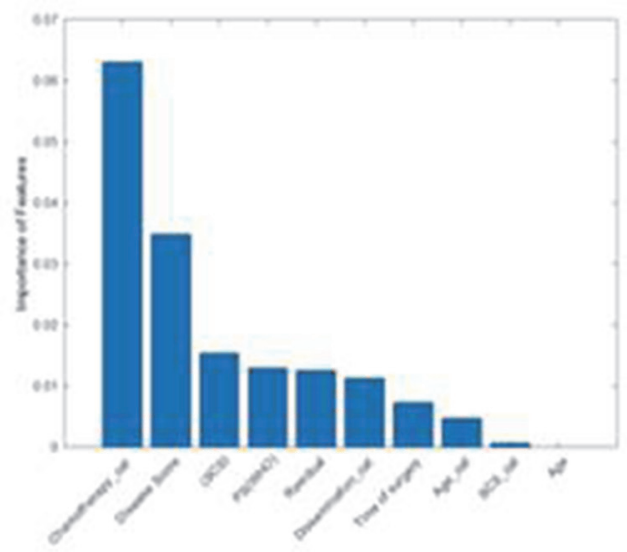

Rank features for classification usin:

minimum redundancy maximum relevance (MRMR) a'gorithm

\section{Abstract 332 Figure 1}

\section{2-year Overall survival}

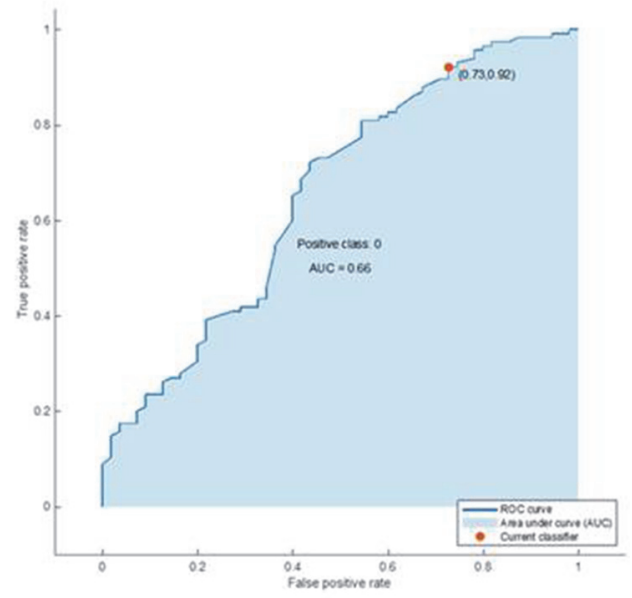

Abstract 332 Figure 2

were considered in the negative class. The study was restricted to the most common prognostic variables and focused on predictive model comparisons. Dataset was split into training and test cohorts with repeated random sampling until there was no significant difference $(p=0.20)$ between the two cohorts with respect to all variables.

Results 172 out of 209 patients with fully curated data were eligible for 2-year prognosis prediction analysis. 104/172 $(60 \%)$ and 55/172 (32\%) patients had disease recurrence or died of disease within two years, respectively. The variable importance for the 2-year progression free survival (PFS) and overall survival (OS) is shown in figure 1. A combination of good performance status, upfront cytoreduction and increased surgical complexity score best predicted 2-year PFS with an accuracy of $63 \%$ and $62.1 \%$ for the SVM and K-NN classifiers, respectively. SVM best predicted 2-year OS by a combination of Carboplatin/Taxol chemotherapy, low disease score, no residual disease, increased surgical complexity score, and upfront cytoreduction with an accuracy of $71.6 \%$ ( AU-ROC: 0.66) (figure 2).

Conclusion ML appears to be promising for accurate estimation of HGSOC prognosis. We provide evidence as to what combination of prognosticators leads to the largest impact on the HGSOC two-year prognosis. The cohort is currently expanding to further examine the short term vs long term contribution of the clinical variables from the comparative models

Disclosures No disclosures.

\section{THE EFFECT OF ENDOMETRIOSIS ON THE PROGNOSIS OF OVARIAN CLEAR CELL CARCINOMA: THE JURY IS STILL OUT}

${ }^{1}$ Felicia Elena Buruiana, ${ }^{1}$ Anastasios Tranoulis, ${ }^{2}$ Bindiya Gupta, ${ }^{1}$ Janos Balega, ${ }^{1}$ Kavita Singh. 'Panbirmingham Gynaecological Cancer Centre, Birmingham City Hospital; Gynaecological Oncology; ${ }^{2}$ The Pan-Birmingham Gynaecological Oncology Centre, City Hospital; Panbirmingham Gynaecological Cancer Centre, Birmingham City Hospital; Gynaecological Oncology

\subsection{6/ijgc-2020-ESGO.122}

Introduction/Background Women with endometriosis carry an increased risk for ovarian clear cell ovarian carcinoma (OCCC), also referred to as 'endometriosis-associated ovarian carcinoma'. Considered as the precursor lesion of a subset of OCCC, the prognostic role of endometriosis amongst women with OCCC remains a field of contention. Few studies have evaluated the prognostic significance of the concurrent endometriosis with conflicting results. The aim of this study was to ascertain the effect of endometriosis on the prognosis of OCCC.

Methodology This was a retrospective cohort study, from 2000 to 2019. Population-based prospectively collected data on OCCC with or without concurrent endometriosis were retrieved via the Pan-Birmingham Gynaecological Oncology database. Ninety-four women with a primary diagnosis of OCCC have been divided into groups based upon the detection of cancer arising from ovarian endometriosis $(n=48$, 